\title{
Der Begriff der Existenz bei Jaspers und Sartre ${ }^{1}$
}

\author{
Csaba Olay
}

\begin{abstract}
This paper investigates the central concept of existence in the work of Jaspers and Sartre. While Jaspers describes the human mode of being in terms of different layers, Sartre sees human existence as a permanent struggle with contingency that should be understood as radical freedom.
\end{abstract}

Im Folgenden wird der Versuch unternommen, den Zentralbegriff der Existenz im Denken von Jaspers und Sartre in einem ersten Schritt für sich genommen zu skizzieren und in einem zweiten Schritt aufeinander $\mathrm{zu}$ beziehen. Für Letzteres ergibt sich bereits ein Anknüpfungspunkt im Denken von Jaspers selbst, da Jaspers seine Existenzphilosophie deutlich vom Existentialismus Sartres abgrenzt. Über die expliziten Bezugnahmen hinaus sollen auch implizite Gemeinsamkeiten und Unterschiede zur Geltung kommen. Als Leitfaden im gegenwärtigen Kontext dient vor allem die Frage, wieweit die beiden Denker, auf übereinstimmende oder abweichende Weise, zur Klärung unserer Seinsweise, also zur Klärung des spezifischen Seins des Einzelnen, beigetragen haben. Den Bezugspunkt der Darstellung bildet im Falle von Jaspers vor allem das Hauptwerk Philosophie, während für die Sartresche Position in erster Linie Das Sein und das Nichts herangezogen wird. ${ }^{2}$ Es lassen sich gleich am Anfang die Interpretationsthesen, die meine Darstellung strukturieren, vorwegnehmen: 1. Im Anschluss an Kierkegaard arbeitet Jaspers ein Stufenmodell menschlicher Seinsweise aus, deren ungegenständlichen Kern die auf eine Transzendenz angewiesene Existenz bildet. 2. Sartre bestimmt dagegen das Grundgeschehen menschlichen Seins als Kontingenzbewältigung, die als eine eigentümliche, radikale Freiheit verstanden wird.

\section{Jaspers}

Die Behandlung von Jaspers' Auffassung wird im Folgenden auf die Frage des eigentümlichen Menschseins fokussieren, deren weiterer Kontext im Zusammenhang seines Denkens bleibt am Rande. Deswegen konzentriert sich die gegenwärtige Darstellung auf die erste Phase von Jaspers' Denkweg (etwa bis 1945) und nimmt als Leitfaden das Problem der spezifischen Seinsweise des Menschen, ohne zu unterstellen, dies sei das Grundproblem von Jaspers gewesen. Der Verzicht auf diese Annahme empfiehlt sich unter anderem deswegen, weil im

\footnotetext{
${ }^{1}$ Eine frühere Fassung dieses Beitrags wurde am 5. Internationalen Klingenthal-Symposium über Karl Jaspers vorgetragen. Den Veranstaltern des Symposiums und den Diskussionsteilnehmern danke ich für die Anregungen, wobei ich insbesondere Anton Hügli und Manuela Hackel verpflichtet bin. Der Aufsatz wurde in der MTA-ELTE Forschungsgruppe Hermeneutik ausgearbeitet und von einem Bolyai Forschungsstipendium der Ungarischen Akademie der Wissenschaften sowie vom Projekt 100922 des ungarischen Wissenschaftlichen Landesforschungsfonds (OTKA) gefördert.

${ }^{2}$ Damit wird in beiden Fällen von einer Binnendifferenzierung des Denkweges abgesehen. Ausgeklammert wird bei Jaspers die Spätphase seines Denkens etwa nach 1945 mit der Zentralstellung der Vernunft und der Beschäftigung mit politischen Fragen, und bei Sartre wird von seiner zweiten, marxistischen Denkperiode abstrahiert. Auf die Frage, ob und in welchen Hinsichten Sartre mit seinen späteren philosophischen Arbeiten das frühere Konzept der Existenz modifiziert bzw. aufgegeben hat, kann nicht eingegangen werden. Das sollte nicht verhindern, eventuelle spätere Überlegungen der Philosophen, die auf Schwachstellen des Existenzbegriffs hindeuten, mit einzubeziehen.
} 
Zentrum der Philosophie von Jaspers die Frage nach dem Sein steht, aber nicht im Sinne einer Ontologie, die mit einer festgelegten Begrifflichkeit verschiedene Seinsweisen zu unterscheiden und in einem abgeschlossenen System zu präsentieren suchte. Im Gegensatz dazu sollte die Philosophie, wie Jaspers sie versteht, das Ganze des Seins in einer prinzipiell offenen Erörterung entfalten. Allerdings erhält man mit dieser Beschreibung kein Kriterium oder keine Definition der Philosophie, mithilfe dessen sie von anderen Bereichen abgegrenzt werden könnte. Es lässt sich aber auf Situationen verweisen, die durch das Fraglichwerden von Gewissheiten zur Philosophie motivieren können: Staunen, Zweifel und Grenzsituationen. Selbstverständlich münden diese intellektuellen Zustände nicht automatisch im Philosophieren; sie können nach einem eigentümlich gestörten Innehalten auch spurlos wieder verschwinden. Jaspers grenzt innerhalb der Philosophie drei Bereiche voneinander ab, in die sich ihm zufolge das Sein selbst gliedert: „das All, das Ursprüngliche und das Eine: das All des Daseins ist Welt, unsere Ursprünglichkeit Existenz, das Eine Transzendenz."3 Die entsprechende Erörterung dieser Bereiche markiert zum einen den Weg der Weltorientierung ${ }^{4}$, zum anderen den der Existenzerhellung und schließlich den der Metaphysik. An dieser unsystematischen Systematik ist auch ersichtlich, dass Jaspers zu Recht meint, in seiner Philosophie sei die Existenzerhellung „,ein unerlässliches Moment des Ganzen, aber nicht das Ganze selber“5. Die Einteilung der Bereiche der Philosophie hängt ferner mit Jaspers' Philosophieverständnis eng zusammen. Philosophie hat ihm zufolge grundsätzlich die Aufgabe der Lebensorientierung für den Einzelnen, woraus auch die Ablehnung der Wissenschaft bzw. der wissenschaftlichen Philosophie jeder Art folgt. Wie imposant auch die Ergebnisse der Erfahrungswissenschaften sein mögen, sie sind nicht imstande, dem menschlichen Leben in den wichtigsten Fragen verbindliche Antworten zu geben. Jaspers folgt der berühmten Diagnose Max Webers, der in seinem Vortrag „Wissenschaft als Beruf" nachdrücklich feststellt, die Wissenschaft sei untauglich für die Führung und Orientierung des Lebens.

Im fast gleichzeitig erschienenen Buch Die geistige Situation der Zeit fasst Jaspers sein Bestreben unter dem Titel Existenzphilosophie wie folgt zusammen:

Existenzphilosophie ist das alle Sachkunde nutzende aber überschreitende Denken, durch das der Mensch er selbst werden möchte. Dieses Denken erkennt nicht Gegenstände, sondern erhellt und erwirkt in einem das Sein dessen, der so denkt. In die Schwebe gebracht durch das Überschreiten aller das Sein fixierenden Welterkenntnis (als philosophische Weltorientierung), appelliert dieses Denken (als Existenzerhellung) an seine Freiheit und schafft den Raum seines unbedingten Tuns im Beschwören der Transzendenz (als Metaphysik). ${ }^{6}$

Dabei fasst philosophische Weltorientierung Jaspers zufolge „nicht letzte Ergebnisse der Wissenschaften zu einem einheitlichen Weltbild zusammen, sondern zeigt die Unmöglichkeit eines solchen gültigen Weltbildes als des einen und absoluten; sie sucht die Fragwürdigkeiten der faktischen wissenschaftlichen Weltorientierung. “7 1937 erklärt Jaspers Existenzphilosophie in den gleichnamigen Groningener Vorlesungen für ,eine Gestalt der einen, uralten Philosophie" ${ }^{\text {*8. }}$.

Jaspers beschreibt das, was er Existenz nennt, im Rahmen vierer Seinsweisen des Menschen, die ein Stufenmodell des Menschseins bilden: Es handelt sich um Dasein, Bewusstsein

\footnotetext{
${ }^{3}$ Jaspers: Philosophie I, S. 28, Herv. i. O.

${ }^{4}$ „Das Wissen von den daseienden Gegenständen heißt Weltorientierung. Es ist nur Orientierung, weil es, stets unabgeschlossen, ein unendlicher Prozeß bleibt, Weltorientierung, weil es ein Wissen von einem bestimmten Sein, nämlich in der Welt wird.“( (ebd., S. 28, Herv. i. O.)

${ }^{5}$ Ebd., S. XXIII.

${ }^{6}$ Ders.: Die geistige Situation der Zeit, S. 145, Herv. i. O.

${ }^{7}$ Ders.: Philosophie I, S. 30, Herv. i. O.

${ }^{8}$ Ders.: Existenzphilosophie, S. 1.
} 
überhaupt, Geist und Existenz. Der Mensch existiert als „empirisches“ oder „,bloßes Dasein“, womit das körperliche, biologische Leben gemeint ist. „Bloßes Dasein“ hat also in erster Linie mit Instinkten, Trieben und Interessen bzw. mit deren Ausleben zu tun. Eine zweite Stufe oder Schicht der menschlichen Seinsweise nennt Jaspers mit einer an Kant erinnernden Formulierung „Bewusstsein überhaupt“. Im Sinn des Verstandes bei Kant meint diese Ebene menschlichen Seins die Fähigkeit und Möglichkeit des logischen Denkens, das allgemeingültig und zwingend funktionieren kann im Gegensatz zum Bewusstsein des bloßen Daseins, das nur intentional auf Zwecke in der Umgebung gerichtet ist. Das Bewusstsein überhaupt beschreibt die Dimension des Menschen als erkennendes Wesen. Die dritte Stufe des Menschseins ist die Ebene des „Geistes“ oder der Vernunft, mit deren Hilfe der Mensch imstande ist, sich auf „geistige Ganzheiten“ zu beziehen. Damit meint Jaspers Wertvorstellungen und Ideen, die in von Menschen gestaltete Sinnzusammenhänge und umfassende Orientierungen eingebettet sind.

Den drei erwähnten Seinsweisen des Menschen ist gemeinsam, dass sie Aspekte des Menschen charakterisieren, die sich empirisch erfassen lassen. Dagegen ist die vierte Seinsweise, die „Existenz" bzw. das ,eigentliche Sein“, nicht empirisch oder rational zu beobachten. Sie bildet die ungegenständliche Seite des Menschen, der im „Aufschwung“ zur Existenz seine individuelle, unvertretbare Art und Weise des Menschseins realisiert. ${ }^{9}$ Der Aufschwung zur Ebene der Existenz geschieht unabhängig vom Willen des Einzelnen und lässt sich nicht objektiv beschreiben, sondern nur in jeweils individuellen eigenen Verwirklichungen erleben. Entsprechend ist ein solcher Aufschwung nicht dauerhaft, vielmehr beschränkt sich er auf existentielle „Augenblicke“. In Klammern gilt es zu bemerken, dass die Eigentlichkeit auch von Heidegger nicht als dauerhafter Zustand, sondern als etwas immer wieder neu zu Gewinnendes konzipiert wird. Die Realisierung der Existenz ist Jaspers zufolge auf zweifache Weise möglich: entweder in Grenzsituationen oder in der eigentlichen Kommunikation, wobei deren Verhältnis in der Jaspers-Forschung umstritten ist. Jaspers kommt also dadurch zur Bestimmung der Existenz, dass er ein nicht objekthaftes Sein nach allem gegenständlich Wissbaren vorfindet: dieses Sein ,bin ich selbst als Existenz. Sie bin ich, sofern ich mir nicht selbst Objekt werde. In ihr weiß ich mich unabhängig, ohne daß ich zu schauen vermöchte, was ich mein Selbst nenne. Aus ihrer Möglichkeit lebe ich; nur in ihrer Verwirklichung bin ich ich selbst. " ${ }^{10}$ Der Wesenskern des Menschseins ist demnach für Jaspers wie auch für Sartre nicht gegenständlich.

Die vergegenständlichende Erfahrung des Alltags muss Jaspers zufolge in die Schwebe gebracht werden, und dazu ist allein die Philosophie fähig, die auf diese Weise auch über die wissenschaftliche Erkenntnis hinausführen kann und muss. Gegenstand dieser Erfahrung ist die Welt als „Inbegriff alles dessen, was mir durch Orientierung des Erkennens als ein zwingend für jedermann wißbarer Inhalt zugänglich werden kann“11; die „Existenz und Transzendenz“, oder, mythisch ausgedrückt, ,Seele und Gott“ gehören nicht mehr in diesen Bereich. Letztere sind nicht im selben Sinne wie die Dinge in der Welt, und dementsprechend können sie auch nicht so gewusst und gekannt werden wie die Dinge. Nach Jaspers ist es für die Grenze der gegenständlichen Erkenntnis charakteristisch, dass sie noch immanent ist und bereits auf Transzendenz hindeutet; derart weist die gegenständliche Erkenntnis über sich hinaus. Das Hinausgehen über das gegenständliche Wissen nennt Jaspers „Transzendieren“, das als ein Überschreiten des gegenständlich Wissbaren in Richtung des Nicht-mehr-gegenständlichWissbaren der Existenz und der Transzendenz gefasst wird. Ohne Transzendieren kann

\footnotetext{
9 Michael Theunissen bemerkt in einer Fußnote seiner Deutung der Verzweiflungsanalyse, dass diese Interpretation sich an die Jasperssche Unterscheidung von Dasein und Existenz anschließt (vgl. Theunissen: Der Begriff Verzweiflung, S. 45).

${ }^{10}$ Jaspers: Philosophie II, S. 1 f., Herv. i. O.

${ }^{11}$ Ebd., S. 1, Herv. i. O.
} 
Philosophie nur begrenzte Gegenstandserkenntnis oder intellektuelle Spielerei sein. Dabei wird der Akzent auf den Vollzug des Überschreitens, und nicht auf die gewonnenen Einsichten gelegt: „Die Wirklichkeit des Philosophierens ist nicht im objektiven Resultat, sondern eine Bewußtseinshaltung “12.

Zwar handelt es sich darum, Existenz und Transzendenz zugänglich zu machen, dies lässt sich jedoch nicht mithilfe des gewöhnlichen Sprechens erreichen. Das sieht man auch daran, dass die Existenz Jaspers zufolge ausdrücklich nicht definiert und erkannt werden kann. Der Ausdruck wird auf folgende Weise erläutert: „Existenz ist, was nie Objekt wird, Ursprung, aus dem ich denke und handle, worüber ich spreche in Gedankenfolgen, die nichts erkennen; Existenz ist, was sich $z$ u sich selbst und darin zu seiner Transzendenz verhält." 13 Jaspers stützt sich in dieser Formulierung zugegebenermaßen auf Kierkegaards Formel vom Selbst in der Krankheit zum Tode. Dass das Sich-zu-sich-selbst-Verhalten mit einem Gottesbezug identifiziert werden kann, war aber bereits bei Kierkegaard eher nur behauptet als begründet: „So ambivalent Kierkegaards Nein zum System ist, so halbherzig ist auch seine Ablehnung philosophischer Gottesbeweise. Insgeheim möchte er die Existenz Gottes aus der Existenz der Verzweiflung beweisen. "14 Zumindest versucht Kierkegaard, die Formel von der Macht, in der das Selbst gründen soll, deskriptiv gar nicht erst einlösen. Immerhin bemüht sich Jaspers, den von ihm im Selbstbezug behaupteten Transzendenzbezug plausibel zu machen, indem er das Erreichen von Existenz als etwas beschreibt, was mir in bestimmten Augenblicken gleichsam „,passiert“. Gleichwohl ist an Jaspers' Erörterung die Vorstellung nicht überzeugend, dass aus dem Umstand, dass ich mich als Selbst vorfinde, folge, dass es eine Macht gibt, die mich gesetzt hat und die dann gleich als Gott verstanden wird. Anders gewendet, die Frage, wer mein Selbst hergestellt hat, ist bereits schief, selbst wenn sie auf exakt diese Weise bereits von Kierkegaard so gestellt wird. ${ }^{15}$

Wie auch immer es mit dem Transzendenzbezug bestellt sein mag, die Aufklärung oder, mit Jaspers' Wort, die „Erhellung“ der Existenz kann nur mittelbar sein; die Philosophie kann nicht eine allgemeingültige Theorie über das Wesen des freien Menschen liefern; sie kann höchstens den Einzelnen zu einem Punkt führen, von dem her er selbst die eigenen Möglichkeiten erfahren muss. Diesen Punkt bestimmt Jaspers nicht ganz eindeutig: Zum einen sind es die Grenzsituationen, zum anderen die existentielle Kommunikation, die die Möglichkeit des Selbstwerdens bieten und gleichzeitig die Unzulänglichkeit des bloß theoretischen Denkens zeigen. Das Problem, wie das Selbstwerden in Grenzsituationen und das Selbstwerden in existentieller Kommunikation sich aufeinander beziehen lassen, sei hier ausgeblendet. ${ }^{16}$

Existenz ist für Jaspers gleichbedeutend mit Selbstsein, wobei dies nicht passiv verstanden werden darf, sondern als eine nicht von jedem realisierte Form menschlichen Lebens. Die Existenz bezeichnet dementsprechend die Aufgabe und die Wahl, im Dasein selbst zu werden und sich nicht in der bloßen Daseinsweise der Menge zu verlieren: „Nicht mein Dasein also ist Existenz, sondern der Mensch ist im Dasein mögliche Existenz. "17 „Dasein“ benennt den Menschen in seinem geschichtlich empirischen Bestand, wie er zumeist, nämlich aufgelöst in der Menge, vorkommt. Jaspers geht so weit, die menschliche Seinsweise, welche

\footnotetext{
${ }^{12}$ Ders.: Philosophie I, S. 264, Herv. i. O.

${ }^{13}$ Vgl. ders.: Philosophie II, S. 15.

14 Theunissen: Der Begriff Verzweiflung, S. 25.

${ }^{15}$ Für eine ausführliche Diskussion der Frage vgl. Evans: „Who Is the Other in The Sickness unto Death?“ und Hannay: „, ,Spirit“ and the Idea of Self as a Reflexive Relation“.

${ }^{16}$ Vgl. dazu Salamun: Karl Jaspers, S. 54 ff: Mit „,dem Konzept der Grenzsituation und mit dem Konzept der existentiellen Kommunikation [sind] doch zwei unterschiedliche Vorstellungen menschlicher Selbstverwirklichung gemeint“" (ebd., S. 55), d.h. die Grenzsituation ist weder als eine Vorstufe der existentiellen Kommunikation noch als mit ihr identisch zu betrachten.

${ }^{17}$ Jaspers: Philosophie II, S. 2.
} 
Selbstständigkeit vermeidet, in Vergnügungen zerstreut ist und sich in gewöhnlichen

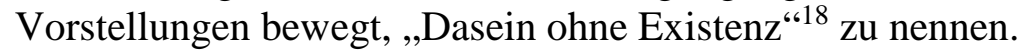

Um sich selbst zu finden und werden zu können, ist man auf eigentümliche Situationen angewiesen, in denen man sich auf besonders intensive Weise selbst begegnet. Zwar ist das menschliche Sein grundsätzlich immer situativ, „ein Sein in Situation“, denn ich kann ,niemals aus der Situation heraus, ohne in eine andere einzutreten "19. Die Situationen des Lebens lassen sich aber mehr oder weniger durchschauen, auch wenn sie mannigfaltig und einzigartig sind. Mit den Grenzsituationen, unter denen Jaspers Tod und Leiden, Kampf und Schuld anführt, hat es eine andere Bewandtnis. Diese Situationen ,sind nicht überschaubar; in unserem Dasein sehen wir hinter ihnen nichts anderes mehr. Sie sind wie eine Wand, an die wir stoßen, an der wir scheitern. Sie sind durch uns nicht zu verändern, sondern nur zur Klarheit zu bringen [...]. Sie sind mit dem Dasein selbst. "20 Die grundlegende Bedeutung dieser Situationen beruht darauf, dass sie einen vor die Alternative stellen, entweder die Augen vor ihnen zu verschließen oder bewusst in sie einzutreten. Letzteres ist Jaspers zufolge die Möglichkeit, man selbst zu werden, weswegen es auch heißen kann: „Grenzsituationen erfahren und Existieren ist dasselbe. “21

Die andere wesentliche Weise des Selbstwerdens erblickt Jaspers, wie erwähnt, in der existentiellen Kommunikation, deren Stellenwert durch den Gedanken bestimmt ist, dass man nicht imstande ist, in der Vereinzelung sich selbst zu finden: „Ich kann nicht ich selbst werden, ohne in Kommunikation zu treten, und nicht in Kommunikation treten, ohne einsam zu sein" ${ }^{\text {"22. }}$. Der zweite Teil des Satzes bezieht sich selbstverständlich nicht auf den Vollzug der Kommunikation, sondern stellt eine Bedingung der Kommunikation als Fähigkeit zur selbstständigen Reflexion und als Notwendigkeit der Stellungnahme dar. Wichtiger noch im vorliegenden Kontext ist der Gedanke, dass die Verwirklichung der Existenz von der Kommunikation als ihrem einzig möglichen Ort abhängig gemacht wird. ${ }^{23}$ Nicht ein einsames Tun, sondern Kommunikation mit Anderen ermöglicht das Selbstwerden, das über die Kommunikation auch mit dem Dasein Anderer verschränkt wird. Jaspers sieht das eigentliche Selbstwerden mit einem Offenbarwerden gegenüber sich selbst und einem anderen Menschen notwendig verbunden. Der springende Punkt dabei ist, dass sich dieses Offenbarwerden nicht isoliert vollziehen lässt, weil ,ich [...] als Einzelner für mich weder offenbar noch wirklich“ bin. Den Prozess dieses Offenbarwerdens in der Kommunikation bestimmt Jaspers als „liebende[n] Kampf ${ }^{\prime 24}$.

Die von Jaspers differenzierten Formen der Kommunikation zeigen deutlich, wie fundamental der Andere für die jeweilige Existenz ist. Er unterscheidet die „Daseinskommunikation“, die sich in objektiven Kategorien wiedergeben lässt, von der „existentiellen Kommunikation“, die eine existentielle Relevanz hat ${ }^{25}$. Zu den objektiven Weisen gehört erstens die Kommunikation in primitiver Gemeinschaftlichkeit, zweitens die Kommunikation in sachlicher Zweckhaftigkeit und Rationalität und drittens die Kommunikation in ideenbestimmter Geistigkeit des Gehalts. Diese Formen entsprechen den drei Ebenen menschlicher Seinsweise. Die existentielle Kommunikation ist für Jaspers wesentlich durch eine radikale Offenheit der Beteiligten charakterisiert, die ihr wechselseitiges Offenbarwerden ermöglicht: „In diesem Kampf wagen

\footnotetext{
${ }^{18}$ Ders.: Die geistige Situation der Zeit, S. 37.

${ }^{19}$ Ders.: Philosophie II, S. 203, Herv. i. O.

${ }^{20}$ Ebd. , Herv. i. O.

${ }^{21}$ Ebd., S. 204.

${ }^{22}$ Ebd., S. 61. Auf die von Jaspers nicht aufgelöste Spannung zwischen Grenzsituation und existentieller Kommunikation hat u.a. Kurt Salamun hingewiesen (Salamun: Karl Jaspers, S. 54 ff.).

${ }^{23}$ Vgl. Jaspers: Philosophie II, S. 242: „Existenz [verwirklicht] sich nur in Kommunikation“.

${ }^{24}$ Ebd., S. 65

${ }^{25}$ Vgl. ebd., S. 54 ff.
} 
beide rückhaltlos sich zu zeigen und infragestellen zu lassen.“26 Ein weiterer Aspekt dieser Offenheit lässt sich als Absage an strategisches Verhalten, etwa durch Maskierungen, aufgenommene Rollen usw., fassen. Gleichzeitig sind die beiden Teilnehmer der existentiellen Kommunikation unvertretbar, und diese Kommunikation als liebender Kampf zweier möglicher Existenzen entwickelt sich im Dialog und zielt auf das Offenbarwerden beider. Selbst wenn der Ausdruck ,existentielle Kommunikation“ die Bedeutung dieses Prozesses für die jeweilige Existenz betonen soll, lassen sich inhaltliche Kriterien dafür kaum feststellen, was „existentiell“ hier bedeutet. ${ }^{27}$ Auch die Bestimmung „liebender Kampf“" hilft nicht bei der inhaltlichen Erläuterung, da sie die Begegnung ihrem Vollzug und ihrer Qualität nach charakterisiert. Man könnte versuchen, die Rede von existentieller Kommunikation eher formal, als eine Weise des Kommunizierens aufzufassen, die die Diskussion an die eigene Existenz „heranlässt“. Damit werden aber andere Formen der Kommunikation nicht von der existentiellen abgegrenzt, weil dies kein hinreichendes Kriterium ist, wenn die Kommunikationspartner nicht für austauschbar gehalten werden, wie im Falle der Daseinskommunikation. ${ }^{28}$

\section{Sartre}

Bei der Darstellung der Grundgedanken von Sartre soll von vornherein betont werden, dass seine schriftstellerische Tätigkeit weitgehend ausgeklammert wird in der Annahme, dass seine Auffassung menschlicher Existenz aus seinen philosophischen Schriften deutlich genug entwickelt werden kann und nicht auf eine Deutung seiner Dramen und Romane angewiesen ist. Ferner soll auch vorausgeschickt werden, dass der Ausdruck „Existenz“ sich nur mit Einschränkungen als Zentralbegriff des Denkens von Sartre verstehen lässt - im Gegensatz zu Jaspers, für den Existenz - Sein - Transzendenz als Hauptgesichtspunkte seiner Philosophie angesehen werden können. Sartre stellt den Terminus „Existenz“ nicht explizit in den Mittelpunkt seines Hauptwerks Das Sein und das Nichts, sondern nur implizit, soweit es ihm um die eigentümliche Seinsweise des Menschen geht. Dies bedeutet nicht, dass die Hinweise auf den Existenzbegriff fehlen, wie z.B. in der Einleitung von Das Sein und das Nichts, wo Sartre an mehreren Stellen Bestimmungen Heideggers über das Dasein auf das Bewusstsein bezieht. Ferner ist der Ausdruck „réalité-humaine“ ein Übersetzungsversuch oder eine Umformulierung von Heideggers „Dasein“, selbst wenn er mit sehr abweichenden Nebenbedeutungen belastet ist. Andererseits haben die Begriffe der Freiheit und des Bewusstseins im Denken von Sartre eine eminentere Stellung als der der Existenz, und damit verbindet sich zugleich ein Hauptmerkmal der Existenz, wie der französische Philosoph sie versteht, nämlich die Freiheit.

Es lohnt sich zunächst, einen kurzen Blick auf die Hauptthemen der vier Teile des Werkes zu werfen. Der erste Teil ist dem Problem des Nichts gewidmet, das mit Hilfe einer eigentümlichen Deutung des Bewusstseins aufgrund seiner Fähigkeit des Nichtens erläutert wird. Im zweiten Teil steht das Bewusstsein, also das Für-sich-sein, mit seinen charakteristischen Leistungen im Mittelpunkt; es werden die Faktizität, die Zeitlichkeit und die Transzendenz erörtert. Sartre behandelt im dritten Teil das Für-Andere-sein, also das Problem der Intersubjektivität, das sowohl den Leib als auch emotionale und soziale Beziehungen zu Anderen betrifft. Der vierte Teil sollte den Übergang von der Ontologie zur Moralphilosophie ermöglichen, und zu diesem

\footnotetext{
${ }^{26}$ Ebd., S. 65.

27 Deshalb ist es ein Missverständnis, wenn Y. Örnek den Vollzug der existentiellen Kommunikation im Philosophieren lokalisieren will (vgl. Örnek: Karl Jaspers, S. 53).

${ }^{28}$ Dies wurde im Einzelnen in meinem Aufsatz „Jaspers und Gadamer über Kommunikation und Dialog“ ausgeführt.
} 
Zweck diskutiert Sartre in diesem Teil die Freiheit in ihrem Verhältnis zur Situation, die Struktur der Handlung und die existentielle Psychoanalyse. Wie aus dieser groben Skizze ersichtlich ist, sind die Schlüsselbegriffe der im Untertitel des Hauptwerkes versprochenen phänomenologischen Ontologie Sartres der Begriff des Bewusstseins und der eines dem Bewusstsein gegenüberstehenden Bereichs, die Sartre Für-sich-sein (pour-soi) und An-sichsein (en-soi) nennt. Die wichtigsten Bestimmungen des Bewusstseins werden von Sartre zunächst mit Blick auf das Verhältnis des Für-sich-seins zum An-sich-sein erörtert, und erst dann ergänzt mit dem Verhältnis des Für-sich-seins zu den Anderen, weil das Für-sich-sein auf das Für-Andere-sein verweist. ${ }^{29}$

Im Zentrum des Hauptwerks steht das Begriffspaar An-sich (en-soi) und Für-sich (pour-soi), wobei bereits die Wahl dieser an Hegel erinnernden Ausdrücke den Anschluss an die Unterscheidung Subjekt-Objekt oder Bewusstsein-Gegenstand andeutet. Gleichwohl sollte nicht übersehen werden, dass Sartre deutlich andere Akzente setzt als Hegel. Die Region des An-sich-seins sei selbständig, geschlossen, undurchdringlich, wohingegen die des Für-sichseins veränderlich, temporal sei. In der Einleitung versucht Sartre zu zeigen, dass die beiden Pole aufeinander angewiesen sind und sich gleichzeitig aus dem je anderen nicht ableiten lassen. Das Phänomen kann nicht auf das Wahrgenommen-Werden (percipi) reduziert werden, und das Bewusstsein ist immer mehr als die ihm erscheinenden Phänomene, es transzendiert die Phänomene. Im Bewusstsein findet sich kein selbstidentisches Ich; sein kontingentes, vorübergehendes Sein lässt sich nur als der Gegensatz zum Sein, also als Nichts bestimmen. Mit den ontologischen Charakterisierungen der beiden Pole des Gegensatzes erhält man auch den Titel des Werks: es geht für Sartre um das Sein und das Nichts. Dabei gilt für das Sein: „Tatsächlich ist das Sein sich selbst opak, eben weil es von sich selbst erfüllt ist. Das drücken wir besser aus, wenn wir sagen, das Sein ist das, was es ist. ${ }^{\text {"30 }}$ Das An-sich wird demnach als eine selbstidentische Massivität beschrieben:

Das An-sich hat kein Geheimnis: es ist massiv. [...] Die Übergänge, das Werden, alles, was die Aussage zuläßt, das Sein sei noch nicht das, was es sein wird, und es sei schon das, was es nicht ist, all das ist prinzipell ausgeschlossen. [...] Es ist unbestimmt es selbst, und es erschöpft sich darin, es zu sein. [...] Das An-sich-sein ist nie möglich oder unmöglich, es ist. ${ }^{31}$

Das An-sich ist von sich selbst voll, und man kann sich keine totalere Fülle, keine vollkommenere Adäquation von Enthaltenem und Enthaltendem vorstellen: es gibt nicht die geringste Leere im Sein, den kleinsten Riß, durch den das Nichts hineingleiten könnte. ${ }^{32}$

Trotz der suggestiven Formulierungen sollte hier die Rückfrage nicht ausbleiben, wie eigentlich das unbestimmte Selbstsein des An-sich verstanden werden könnte, da ein Ding, wenn es es selbst sein soll, keineswegs unbestimmt bleiben kann. Es gehört zum Sinn des „Es-selbstBleibens", dass darin eine Bestimmtheit impliziert ist. Das Ding selbst, und nichts anderes, soll ja bleiben, was und wie es ist. Als kleine Fußnote könnte man ferner hinzufügen, dass Aristoteles im siebten Buch (Z) der Metaphysik den Gegensatz von Dynamis und Energeia eben deswegen einführt, damit gesagt werden kann, etwas sei der Möglichkeit nach etwas, was es der Wirklichkeit nach noch nicht ist. Die spärlichen Bestimmungen des dem Bewusstsein entgegengesetzten Bereichs sind letzten Endes alles andere als unproblematisch in der Sartreschen Ontologie.

Im Gegensatz zum An-sich-sein charakterisiert Sartre in der Bestimmung des Für-sich-seins (pour-soi) das Sein des Bewusstseins mit der berühmt gewordenen Formel ,als das seiend, was

\footnotetext{
${ }^{29} \mathrm{Vgl}$. Sartre: L'être et le néant, S. 267.

${ }^{30}$ Ders.: Das Sein und das Nichts, S. 42, Herv. i. O.

${ }^{31}$ Ebd., S. 43 f., Herv. i. O.

${ }^{32}$ Ebd., S. 165.
} 
es nicht ist, und nicht das seiend, was es ist ${ }^{\star 33}$. Zwar erinnert die Formel an Hegels Bestimmung der Zeit aus der Enzyklopädie: ,Sie ist das Sein, das, indem es ist, nicht ist, und indem es nicht ist, $i s t^{\text {*34 }}$. Aber sachlich aufschlussreicher ist es meines Erachtens, die Formulierung auf eine von Sartre nicht explizit gemachte, prominente Stelle in Sein und Zeit zu beziehen, die unser Dasein als Möglichsein charakterisiert. ${ }^{35}$ Führt man die bekannte Formel auf Heideggers Idee zurück, ist sie offensichtlich mit Zeitausdrücken zu ergänzen: ,(noch) nicht sein, was es ist, und sein, was es (noch) nicht ist“. Die Zeitbestimmung ist hier fundamental: Man ist nicht im Allgemeinen, was man nicht ist, sondern nur in ganz bestimmtem Sinne innerhalb eines zeitlichen Ablaufs. Man ist tatsächlich noch nicht, was man plant zu werden. Was Sartre als eigentümliches Seinsverhältnis ausgeben möchte, betrifft im Wesentlichen die Frage, wie Absichten, Pläne und Projekte zum Menschen (zu seinem Sein) gehören. ${ }^{36}$

Die Grundbestimmung des Bewusstseins ist die Negation, verstanden als ein aktiver Bezug zum Nichts, die in allen wesentlichen Dimensionen des menschlichen Seins zum Tragen kommt. ${ }^{37}$ Diese herausgehobene Stellung der Negation wird auch dadurch unterstrichen, dass in der Entwicklung des Gedankenganges von der Negation ausgegangen wird und erst von ihr her die Freiheit - und auch die Unaufrichtigkeit (mauvaise foi) - erläutert und eingeführt wird ${ }^{38}$. Sie bildet auch gewissermaßen die Grundstruktur des Für-sich, weil das Subjekt sich durch die Negation auf anderes als es selbst bezieht. Sartre gründet seine Argumentation auf der Negation (im Sinn von verneinenden Sätzen) und auf der Imagination, die ihm zufolge Aspekte der nichtenden Tätigkeit (activité néantisante) sind. Durch die Betonung der Ungebundenheit löst Sartre auch die enge Verbindung zwischen der Ungebundenheit im Sinne von Willkür und Autonomie im Sinne von Selbstgesetzgebung, wie sie für den Deutschen Idealismus charakteristisch war, auf.

Ein weiteres wesentliches Merkmal des Bewusstseins ist die Intentionalität, die Sartre mit eigenwilligen Akzentuierungen von Husserl übernimmt, und das gibt auch Anlass zu klären, warum der Grundgegensatz en-soi und pour-soi irreführend ist. Dieses Vokabular verdeckt die tiefgreifende Umformung des Verhältnisses von Subjekt und Objekt, die in der Phänomenologie vollzogen wurde: Bei Hegel ist die Intentionalität noch nicht vorhanden, sondern es wird davon ausgegangen, dass das Subjekt und das Objekt nur für ein alltägliches Denken getrennt zu sein scheinen, während aus der Perspektive der philosophischen Spekulation ihre Identität, die beide mitsamt ihrer Differenz umfasst, einsichtig gemacht werden kann. Verwirrend ist nun der Gebrauch, den Sartre von der im Grunde monistischen Idee der Intentionalität macht, indem er diese eindeutig in eine dualistische, und zwar starr kartesianische Richtung überschreitet. Ähnliches gilt für Sartres Anlehnung an Hegel, die sicherlich im Gegensatz zu seinem Kartesianismus und Dualismus eine Identitätsphilosophie heraufbeschwört. Sartre ergänzt nun das Gerichtetsein des Bewusstseins auf ein Objekt mit

\footnotetext{
${ }^{33}$ Ebd., S. 42.

${ }^{34}$ Hegel: Enzyklopädie, § 258, S. 48, Herv. i. O.

35 „Auf dem Grunde der Seinsart, die durch das Existenzial des Entwurfs konstituiert wird, ist das Dasein ständig ,mehr', als es tatsächlich ist, wollte man es und könnte man es als Vorhandenes in seinem Seinsbestand registrieren. Es ist aber nie mehr, als es faktisch ist, weil zu seiner Faktizität das Seinkönnen wesenhaft gehört. Das Dasein ist aber als Möglichsein auch nie weniger, das heißt das, was es in seinem Seinkönnen noch nicht ist, ist es existenzial.“ (Heidegger: Sein und Zeit, S. 145, Herv. i. O.)

${ }^{36}$ Biemels treffender Kommentar dazu lautet wie folgt: „Bewußtsein ist nicht nur das, was es wirklich ist. [...] Dabei bezieht sich das Nicht [...] nicht etwa auf nichtbewußtseinsmäßig Seiendes, sondern auf Seinsmöglichkeiten, die dem Bewußtsein offen sind. [...] In seinen Möglichkeiten übersteigt es jeweils das faktisch Gegeben-sein. Der Gegenbegriff zur Faktizität tritt hier ins Spiel, nämlich der Begriff Transzendenz." (Biemel: Sartre, S. 65, Herv. i. O.)

37 ,Sartre pose un néant qui ne se réduit pas à la négation, mais révèle l'individu existant dans sa totalité. L'activité néantisante instaure la dynamique du pour-soi et de l'en-soi. Elle fonde la définition de la conscience à travers son manque essentiel.“ (Wald Lasowski: Jean-Paul Sartre, S. 37)

${ }^{38} \mathrm{Vgl}$. Sartre: L'être et le néant, S. 111.
} 
einem präreflexiven Cogito, das als Bedingung des kartesianischen Cogito fungiert. ${ }^{39}$ Diese eigentümlichen Akzentuierungen können mit ein Grund sein, weswegen Sartre oft nur mit Vorbehalt für einen Phänomenologen gehalten wird. ${ }^{40}$ Die Einführung der Unterscheidung zwischen dem Sein des Phänomens und dem Phänomen des Seins lässt sich im Anschluss an Husserl nicht überzeugend aufrechterhalten. Allerdings deutet Sartre den Gedanken der Intentionalität im Rückgriff auf seine früheren Arbeiten in ein Argument für die menschliche Freiheit um: Um etwas vorstellen zu können, muss das Bewusstsein fähig sein, sich von der Welt losreißen zu können, auf die es intentional gerichtet ist. Im Sinne der Frühschrift L'imagniaire wird die Freiheit des Bewusstseins über das Imaginäre aufgezeigt, und zwar mit einem negativistischen Akzent, da die Freiheit vor allem als ständiges Überschreiten des sinnlich Gegebenen gedeutet wird.

Eine Konsequenz der Vorstellung von einer derart ins Bewusstsein eingebetteten Freiheit ist, dass sie mit dem Menschsein zusammenfällt:

Wir haben übrigens schon darauf hingewiesen, daß das Verhältnis der Existenz zum Wesen beim Menschen nicht dem gleicht, was es für die Dinge der Welt ist. Die menschliche Freiheit geht dem Wesen des Menschen voraus und macht dieses möglich, das Wesen des menschlichen Seins steht in seiner Freiheit aus. Was wir Freiheit nennen, ist also unmöglich vom Sein der ,menschlichen-Realität‘ zu unterscheiden. Der Mensch ist keineswegs zunächst, um dann frei zu sein, sondern es gibt keinen Unterschied zwischen dem Sein des Menschen und seinem ,Freisein $^{\circ 41}$.

Sartre thematisiert nicht, oder zumindest nicht nur die positive Seite der Freiheit, sondern auch ihren Lastcharakter, der an einigen Stellen deutlich zutage tritt: ,,ich bin verurteilt, frei zu sein. “42

Zumindest hinweisen sollte man an dieser Stelle auf eine Grunderfahrung, von der her Sartre die spezifische menschliche Existenz deutet, nämlich auf die Kontingenz. Sartre erfasst Kontingenz als den Umstand, sich auf unableitbare und darum zufällige Weise in einer opaken, stabilen Umgebung zu finden. Das Problem, ob auf diese Erfahrung der von Sartre beschriebene „Ekel“ die allgemeine Reaktion ist, sollten wir zunächst ausblenden, unter anderem deswegen, weil diese Reaktion übertrieben zu sein scheint. Die Betonung der Kontingenz sollte vielmehr als implizite Grundlage des Freiheitbegriffes von Sartre verstanden werden, dessen zentrale Stelle leicht das bestimmende Motiv der Kontingenz verdeckt. Das Konzept der Existenz als Freiheit bei Sartre betrifft demnach, genauer betrachtet, Existenz als Ertragen-Müssen der Kontingenz und die Suche nach Mitteln, sie zu überwinden. Ob es den „Ekel“ gibt, ob wir uns wirklich in Situationen vorfinden, die mit einer derart starken Reaktion treffend beschrieben werden, ist eine Frage für die kritische Auseinandersetzung mit Einzelheiten in Sartres Werk. Wie bereits erwähnt, ergänzt Sartre das Für-sich-sein ganz entschieden und nicht nur im nachträglichen Sinne mit einem Für-Andere-Sein: ,ihr [der menschlichen-Realität] Für-sich-

\footnotetext{
39 ,[J]edes objektsetzende Bewußtsein ist gleichzeitig nicht-setzendes Bewußtsein von sich selbst.“ (ders.: Das Sein und das Nichts, S. 21); ,das nicht-reflexive Bewußtsein ermöglicht erst die Reflexion: es gibt ein präreflexives Cogito, das die Bedingung des kartesianischen Cogito ist.” (ebd., S. 22).

${ }^{40}$ Dermot Moran spricht etwa von einem ,undisziplinierten Eklektizismus“ (Moran: Introduction to Phenomology, S. 355) „By the time of Being and Nothingness, however, Sartre considered Husserl to be a phenomenalist who thought of the object as the sum of its appearances, and who tended towards Kantian idealism (BN 73; 111).“ (ebd., S. 359) Siehe auch das einschlägige Kapitel in Waldenfels: Phänomenologie in Frankreich, S. 63-141.

${ }^{41}$ Sartre: Das Sein und das Nichts, S. 84, Herv. i. O.

${ }^{42}$ Ebd., S. 764.

In diesem Kontext wäre die Frage interessant, ob etwas bei Sartre der Analyse des Man bei Heidegger entspricht. Heidegger erblickt im Phänomen ,das Man“ eine stabilisierende Struktur, die unter anderen die Funktion hat, vom radikalen Möglichsein des Daseins zu entlasten.
} 
sein impliziert komplementär dazu ein Für-Andere-sein“433. Mit Blick auf die Intersubjektivität betont Sartre gegen Husserl und Heidegger und im Einklang mit Hegels Dialektik von Herr und Knecht den negativen und konfliktbehafteten Charakter der Beziehung zum Anderen. Von vornherein hebt Sartre den problematischen Charakter des Anderen hervor. Die Begegnung mit dem Anderen bedeutet für ihn immer einen Freiheitsverlust, da sie in Form des ErblicktWerdens erfolgt und mit sich bringt, dass ich vom Anderen zum bloßen Objekt ,verdinglicht“ bzw. auf ein bloßes An-sich-sein reduziert werde. Wenn der Andere mich erblickt, ,nichtet“ er meine Freiheit und beraubt mich meiner Transzendenz, die ich als Subjektivität bin. ${ }^{44}$ Mit Sartres Worten: „Während ich versuche, mich vom Zugriff des Andern zu befreien, versucht der Andre, sich von meinem zu befreien; während ich danach trachte, den Andern zu unterwerfen, strebt der Andre danach, mich zu unterwerfen. “45

Sartre behandelt den Anderen mit Hilfe einer Beschreibung des Blickes (le regard). Die Pointe dieser Perspektive liegt darin, dass dadurch der Andere nicht als ein spezielles Objekt unter anderen gefasst wird, sondern über die Veränderung meiner Selbsterfahrung zur Kenntnis genommen wird. Sartre hebt als maßgebenden Zug der Begegnung mit dem Anderen das DemBlick-des-Anderen-ausgeliefert-Sein hervor - das ist die primäre Perspektive, in die Sartre die Intersubjektivität stellt. Dabei kommt es ihm in erster Linie auf eine Pointe an, die bereits bei Aristoteles eine Rolle spielt: die Spannung zwischen meinem Selbstverständnis und meiner Abhängigkeit von der Beurteilung der Anderen, deren Urteil aus meiner Leistung und meinen Taten nicht automatisch folgt. Diese störende Möglichkeit ist der sachliche Grund, warum Aristoteles die politisch-praktische Lebensweise (bios politikos) niedriger als die theoretische Lebensform einschätzt. Erstere ist weniger unabhängig, weil sie auf das Ansehen (timé) aus ist, und es die Anderen sind, die es geben oder verweigern.

Diese Abhängigkeit vom Anderen wird Sartre zufolge dadurch noch schwerwiegender, dass ich nur über die Anderen zu mir selbst komme; den vergegenständlichenden, objektivierenden Blick auf mich selbst habe ich nur von Anderen, da ich mich nach Sartre nicht selber zum Objekt machen kann. Da scheint mir jedoch eine Hysterisierung der Objektivierung vorzuliegen. Auffälliger Weise fehlt die Berücksichtigung der Möglichkeit des „unparteiischen Beobachters“, also etwa die Annahme eines Normalzuschauers - von der wir tatsächlich ausgehen, wenn wir versuchen, ein „Normalbild“ über uns zu bekommen, da wir nicht jedem trauen, weder jenen, die uns zu wenig, noch jenen, die uns zu sehr lieben. Dieses Vorgehen ist darin mit der existentiellen Kommunikation bei Jaspers verwandt, dass Andere mir über mich selbst Auskunft geben, aber für Jaspers steht existentielle Kommunikation unter Vorbedingungen, wie etwa unter der Bedingung der Offenheit. Das wiederum zeigt, dass nicht jede Begegnung mit einem Anderen ernst genommen und für aufschlussreich gehalten wird.

\section{Schlussbetrachtung}

Jaspers und Sartre stellen, wie gezeigt, den Begriff Existenz auf verschiedene Weise in den Mittelpunkt. Der gemeinsame Grundgedanke beider Denker lässt sich als das Konzept des in

\footnotetext{
${ }^{43}$ Ebd., S. 137.

${ }^{44}$ Vgl. ebd., S. 457-538

${ }^{45}$ Ebd., S. 638.

„Anders als bei Husserl, der Intersubjektivität zur Erklärung der Objektivität heranzieht, wird der Andere durch seinen „Blick“ als Bedrohung und Zerstörung der Eigenwelt des isolierten Subjekts erfahren. Denn im Blick liegt eine fremde Perspektive, ein fremdes Imaginäres, das niemals vollständig mit dem eigenen Horizont zur Deckung gebracht werden kann. Das gilt auch für die Erfahrung des fremden Leibes, der in seiner Konkretheit und Individualität fasziniert, aber auch im Liebesakt keine definitive Verschmelzung gewährt.“ (Fellmann: Phänomenologie, S. 121) Vgl. dazu den Versuch von Steve Martinot, die Sartresche Beschreibung des Blicks als Theorie des Dialogs zu deuten (Martinot: „The Sartrean Account of the Look as a Theory of Dialogue“).
} 
seinem Kern ungegenständlichen Menschseins formulieren: ein Mensch oder ein Selbst zu sein, heißt nicht, ein Gegenstand unter anderen zu sein. Es ist nicht auszuschließen, dass Sartre zu einer nicht kausal determinierten Auffassung des Seelenlebens auch durch die Übersetzungsarbeit an Jaspers' Psychopathologie motiviert gewesen war; selbst wenn es so ist, arbeitet er dennoch eine deutlich andere Variante ungegenständlicher Auffassung von menschlicher Existenz aus. ${ }^{46}$ In der Abgrenzung ihrer Positionen sind in erster Linie folgende Punkte von Bedeutung.

Erstens handelt es sich um eine wesentliche Differenz in der methodologischen Fassung der philosophischen Aufgaben und der angemessenen Sprechweise. Stichwortartig lässt sich diese Differenz als die des appellativen Denkens bei Jaspers und der phänomenologischen Beschreibung bei Sartre fassen, selbst wenn Sartres Zuordnung zur Phänomenologie, wie oben angedeutet, nicht unproblematisch ist. Vielleicht auch mit Blick auf diesen Gegensatz sollte die entschiedene Abgrenzung seiner Existenzphilosophie von dem Existentialismus Sartres bei Jaspers verstanden werden. In einem Brief an Jean Wahl schrieb Jaspers: „L'existentialisme est la mort de la philosophie de l'existence "47, und die markante Unterscheidung von Existentialismus und Existenzphilosophie wird in Vernunft und Existenz wie folgt präzisiert:

Existenzerhellung erkennt nicht die Existenz, sondern appelliert an ihre Möglichkeiten. Sie würde aber als Existentialismus ein Sprechen wie von einem erkannten Gegenstand sein und würde, gerade weil sie der Grenzen innewerden und den unabhängigen Grund erhellen soll, nur um so tiefer in die Irre gehen, indem sie Erscheinungen der Welt unter ihre Begriffe erkennend und beurteilend subsumierte. - In jeder der Verfestigungen, Isolierungen und Verabsolutierungen geht also der eigentliche Gedanke des Umgreifenden verloren. Im gegenständlich gewordenen Umgreifenden ist nicht mehr das wahre Umgreifende. ${ }^{48}$

Dieser Abgrenzungsversuch hängt aber ganz entschieden von der Möglichkeit einer transzendierenden Philosophie mit grundsätzlichem Appellcharakter ab, und in dieser Hinsicht sind mehrere skeptisch. ${ }^{49}$ Ein Grundproblem dieses Denkens liegt sicherlich in der von Jaspers bewusst in Kauf genommenen Ungebundenheit und fehlenden Überprüfbarkeit seiner Sprechweise. Ihm zufolge kann es hier nicht mehr um beweisbare Behauptungen gehen, sondern ausschließlich um Mitteilungen, die auf ein gedankliches Nachvollziehen angewiesen sind. Sicherlich stammt die ambivalente methodische Stellung des Transzendierens aus seiner Verbindung mit der indirekten Mitteilung, deren Idee für Jaspers bereits seit der Psychologie der Weltanschauungen maßgeblich war. Das überaus sympathisierende Porträt der „Propheten indirekter Mitteilung”, die im Gegensatz zu den „Prinzipienlehrer[n]” und den „Totalitätslehrer[n]“ weder versuchen, Regeln der Lebensführung und des Handelns aus Grundsätzen abzuleiten, noch allem seinen Ort zuweisen und ein rationales Gehäuse konstruieren wollen, sondern lediglich darum bemüht sind, aufmerksam zu machen, zu verunsichern und in Unruhe zu versetzen, lässt keinen Zweifel an Jaspers' Präferenzen. ${ }^{50}$

\footnotetext{
${ }^{46}$ An dieser Stelle kann man offenlassen, welche Bedeutung der frühen Bekanntschaft von Sartre mit der Psychopathologie von Jaspers beigemessen wird: „It is of particular interest that while at the École Sartre took a course in pathological psychology, and worked with his friend Paul Nizan on a translation of Karl Jaspers' Allgemeine Psychopathologie [...], which not only underlined the importance of mental disorder for the philosophy of mind and psychology, but also exposed Sartre to a sophisticated formulation of the anti-naturalistic position that the key to psychological exlanation lies in the discovery of non-causal connections of meaning." (Gardner: Sartre's Being and Nothingness, S. 3, Herv. i. O.)

${ }^{47}$ „Der Existentialismus ist der Tod der Existenzphilosophie“ (zit. n. Colette: L'existentialisme, S. 6).

48 Jaspers: Vernunft und Existenz, S. 54, Herv. i. O.

${ }^{49}$ Mit Blick auf das transzendierende Philosophieren spricht etwa Kurt Salamun im Anschluss u.a. an Bollnow von einer „,methodische[n] Sackgasse“ (Salamun: Karl Jaspers, S. 34 f.).

${ }^{50}$ Vgl. Jaspers: Psychologie der Weltanschauungen, S. 376-79. Zu diesem Gegensatz von appellativem Denken und phänomenologischer Beschreibung muss ergänzend hinzugefügt werden, dass Sartre in seinem literarischen
} 
Zweitens lässt sich der inhaltliche Hauptunterschied der beiden Denker in der wesentlich abweichenden Deutung der Freiheit der ungegenständlichen Existenz festlegen: während für Jaspers die Freiheit des Menschen, mit einbegriffen die Möglichkeit des „Aufschwungs“ zur Existenz, sich in stabilen Verhältnissen bewegt (Dasein - Verstand - Geist), verliert für Sartre das radikal mit dem Freisein identifizierte Existieren jeden Anhaltspunkt. In diesem Sinne bildet Existenz für Jaspers zwar eine ausgezeichnete, aber nicht von jedem realisierte oder mobilisierte Schicht unserer Weise zu sein, selbst wenn sich der Einzelne unleugbar am Ideal des zu sich findenden Menschen orientiert. ${ }^{51}$ Dagegen deckt sich Existenz für Sartre mit dem Menschsein schlechthin.

Drittens findet sich ein grundverschiedenes Verhältnis zur Transzendenz bei beiden Denkern. Jaspers erkennt nicht nur die Transzendenz an, er definiert sogar Existenz, wie oben gesehen, zum Teil mit einem Transzendenzbezug. Währenddessen hält Sartre einen Transzendenzbezug nicht nur nicht für wesentlichen Bestandteil der menschlichen Existenz, sondern bestreitet vehement, dass überhaupt ein Bezug zur Transzendenz ausgewiesen werden könnte. Dieser Aspekt bestimmt auch die Beziehung beider Philosophen zu Kierkegaard, dem Jaspers in dieser Hinsicht ausdrücklich folgt. In der Bejahung der Transzendenz als Strukturmoment der Existenz schließt sich Jaspers in einem Punkt nicht an Kierkegaard an, und zwar in der Nachfolge Christi als der einzigen dem Menschen angemessenen Lebensform, die mit Jaspers' entschiedener Abweisung jeder Offenbarung unvereinbar ist. ${ }^{52}$

Viertens haben die Intersubjektivität bzw. die Anderen einen deutlich anderen Stellenwert in den Konzeptionen von Jaspers und Sartre. Die existentielle Kommunikation stellt für Jaspers eine Bedingung des Selbstwerdens dar, und insofern gehört die eigentliche Begegnung mit dem Anderen zu den ausgezeichneten positiven Möglichkeiten menschlicher Existenz. In seinem Konzept des Erblickt-Werdens fasst dagegen Sartre die Rolle der Anderen mit genau umgekehrten Vorzeichen als ernste Gefährdung menschlicher-Realität. Anstelle eines dialogischen Raums für die Offenbarung des Selbst sieht der französische Denker in den zwischenmenschlichen Beziehungen vor allem einen Freiheitsverlust in Form von Verdinglichungs- und Konfliktverhältnissen, deren Beschreibung tief von der Hegelschen HerrKnecht-Dialektik beeinflusst wurde.

Schließlich divergieren beide Autoren im Hinblick auf den gesellschaftlich-geschichtlichen Fortschritt und vor allem auf die Zeitdiagnose. Jaspers war seit seiner Jugend froh, dass ,das Menschengeschlecht [...] nicht von unseren Zeitgenossen allein repräsentiert wird", und angeblich sagte er oft: „Größe ist, was wir nicht sind. “53 Dagegen schätzt Sartre die Gegenwart zumindest nicht schlimmer als frühere Zeiten ein, selbst wenn er die Varianten gesellschaftlicher Unterdrückung und die Möglichkeiten der Einschränkung der Freiheit sensibel beschreibt.

Zusammenfassend lässt sich sagen, dass die verschieden akzentuierten Konzeptionen menschlichen Existierens bei Jaspers und Sartre zwar den gemeinsamen Grundgedanken der Ungegenständlichkeit menschlicher Existenz teilen, gleichzeitig unterscheiden sie sich stark in einigen grundlegenden Punkten. Die hier versuchte Darstellung zielte zum einen auf die Herausstellung dieser Differenzen methodologischer und inhaltlicher Art. Zum anderen wurde der jeweilige systematische Kontext der Analyse der Existenz dargelegt, um auch auf Motivationen und eventuelle Schwachstellen hinzeigen zu können, die eine übergreifende

Schaffen für solche Appelle Ausdrucksmöglichkeiten gefunden hat, die Jaspers mithilfe seines Denkens formulieren wollte.

51 „Das Wunderbare, das einzige eigentliche Seiende, das mir begegnet, ist der Mensch, der er selbst ist“ (ders.: Philosophie II, S. 44, Herv. i. O).

${ }^{52}$ Vgl. ders.: Der philosophische Glaube, S. 516.

${ }^{53}$ Zit. n. Saner: Jaspers, S. 135. 
Theorie menschlicher Existenz, die die Lehre aus dem Werk von Jaspers und Sartre ziehen möchte, berücksichtigen sollte.

\section{LITERATURVERZEICHNIS}

Biemel, Walter: Jean-Paul Sartre. Rowohlt: Reinbek 1965.

Colette, Jacques: L'existentialisme. PUF: Paris 1996.

Evans, C. Stephen: „Who Is the Other in The Sickness unto Death?“. In: ders.: Kierkegaard on faith and the self: collected essays. Baylor University Press: Waco,Texas 2006. S. 263-276.

Fellmann, Ferdinand: Phänomenologie. Junius: Hamburg 2009.

Gardner, Sebastian: Sartre's Being and Nothingness. Continuum: London/New York 2009.

Hannay, Alistair: Kierkegaard and Philosophy. Selected Essays. Routledge: London 2003. S. 64-75.

Hegel, Georg Wilhelm Friedrich: Enzyklopädie der philosophischen Wissenschaften im Grundrisse. Zweiter Teil. Werke. Bd. 9. Suhrkamp: Frankfurt/M 1986.

Heidegger, Martin: Sein und Zeit. 16. Aufl. Max Niemeyer: Tübingen 1986.

Jaspers, Karl: Die geistige Situation der Zeit. de Gruyter: Berlin 1932.

Ders.: Vernunft und Existenz. Batavia J.B. Wolters: Groningen 1935.

Ders.: Existenzphilosophie. 2. Aufl. de Gruyter: Berlin 1956.

Ders.: Psychologie der Weltanschauungen. 6. Aufl. Springer: Berlin/New York 1971.

Ders.: Philosophie, 3 Bde., 4. Aufl. Springer: Berlin/New York 1973.

Ders.: Der philosophische Glaube angesichts der Offenbarung. 3. Auflage. Piper: München/Zürich 1984.

Martinot, Steve: „The Sartrean Account of the Look as a Theory of Dialogue“. In: van den Hoven, Adrian/Leak, Andrew (Hg.): Sartre Today. Berghahn Books: New York/Oxford 2005. S. 43-61.

Moran, Dermot: Introducion to Phenomenology. Routledge: London/New York 2000.

Olay, Csaba: „Jaspers und Gadamer über Kommunikation und Dialog“. Jahrbuch der Österreichischen Karl-Jaspers-Gesellschaft. Jahrgang 24/2011. S. 57-77

Örnek, Yusuf: Karl Jaspers. Philosophie der Freiheit. Alber: Freiburg/München 1986.

Salamun, Kurt: Karl Jaspers. 2. Aufl. Königshausen \& Neumann: Würzburg 2006.

Saner, Hans: Karl Jaspers. Rowohlt: Reinbek 1970.

Sartre, Jean-Paul: Das Sein und das Nichts. Versuch einer phänomenologischen Ontologie. Rowohlt: Reinbek 1998.

Theunissen, Michael: Der Begriff Verzweiflung. Korrekturen an Kierkegaard. Suhrkamp: Frankfurt/M 1993.

Wald Lasowski, Aliocha: Jean-Paul Sartre. Pocket: Paris 2011.

Waldenfels, Bernhard: Phänomenologie in Frankreich. Suhrkamp: Frankfurt/M 1983. 